(2) Open Access Full Text Article

ORIGINAL RESEARCH

\title{
Learning Curve of Botulinum Toxin Bladder Injection for the Treatment of Refractory Overactive Bladder
}

\author{
Marta Barba (D) \\ Tetyana Lazar' \\ Alice Cola' \\ Giuseppe Marino' \\ Stefano Manodoro ${ }^{2}$ \\ Matteo Frigerio $\left.{ }^{3}\right)^{3}$ \\ 'University Milano-Bicocca, San Gerardo \\ Hospital, Monza, Italy; ${ }^{2}$ ASST Santi Paolo \\ e Carlo, San Paolo Hospital, Milano, Italy; \\ ${ }^{3}$ ASST Monza, San Gerardo Hospital, \\ Monza, Italy
}

Correspondence: Marta Barba

University Milano-Bicocca, San Gerardo

Hospital, Via Pergolesi 33, Monza, 20900,

Italy

Tel +390392339434

Email m.barba8792@gmail.com
Purpose: Intradetrusor botulinum toxin injections is an established second-line treatment for patients with refractory overactive bladder syndrome (OAB). We aimed to evaluate the learning curve of intradetrusor injections with botulinum toxin for the treatment of refractory OAB.

Patients and Methods: This retrospective study analyzed all women with idiopathic and refractory $\mathrm{OAB}$ who underwent botulinum toxin bladder injections performed by residents with no previous experience with operative cystoscopy under consultant supervision. Baseline International Consultation on Incontinence Questionnaire-Short Form questionnaire (ICIQ-SF) and Euroqol (EQ-5D) questionnaires were collected. Procedural data (operative time, number of valid injections, complications, subjective easiness, perceived tolerability) and patients' outcomes (Patients Global Impression of Improvement (PGI-I), $\triangle I C I Q-S F$, $\triangle E Q-5 D$, need for self intermittent catheterization, duration of efficacy) were considered as markers to evaluate learning curves for each resident.

Results: Twenty-seven patients underwent intravesical injection of botulinum toxin performed by residents. Only a grade 1 Clavien-Dindo complication occurred, and none of the patients had urinary retention. PGI-I was very satisfactory, scoring $1.4 \pm 0.9$. Both ICIQ-SF and EQ-5D 2-2 were statistically improved ( $p<0.00001$ and $p=0.04$, respectively). The mean duration of efficacy resulted to be $8.0 \pm 4.3$ months. A positive effect of the learning curve was observed only for operative time, subjective easiness, and perceived tolerability. Conclusion: Intradetrusor botulinum toxin injection for the treatment of refractory $\mathrm{OAB}$ is characterized by high efficacy and very low complications even at the very beginning of the learning curve when performed under proper mentorship.

Keywords: botulinum toxin, cystoscopy, learning curve, overactive bladder, urodynamics

\section{Introduction}

OAB syndrome is defined by The International Continence Society (ICS) as "urgency, with or without urge incontinence, usually with frequency and nocturia". ${ }^{1}$ It is a common clinical condition, affecting $16.9 \%$ of women in the United States. $^{2}$ The aetiology of OAB is still unclear, but it has been related to bladder hypersensitivity, low bladder compliance, detrusor overactivity, ${ }^{3}$ or pelvic floor surgery. ${ }^{4,5}$ Detrusor overactivity represents the most common cause of OAB and can be identified in $64 \%$ of patients during urodynamics. ${ }^{6}$ This can be further categorized into two distinct subgroups: either neurogenic or idiopathic (nonneurogenic), according to the presence or not of neurologic diseases or conditions such as 
Parkinson's disease, multiple sclerosis, and/or a spinal cord injury. First-line treatment for idiopathic OAB includes behavioural changes: bladder training and lifestyle modification (decreasing intake of alcohol, caffeine, and acidic and spicy foods). ${ }^{7}$ While a conservative approach is justified initially, pharmacological therapy remains a milestone in the management of women with $\mathrm{OAB}$ and comprehends antimuscarinics and beta- 3 adrenergic receptor agonists. ${ }^{7,8}$ For patients whose OAB symptoms are refractory to conventional medicinal therapy, options include neuromodulation (percutaneous tibial nerve or sacral nerve stimulation), surgical treatment, and intradetrusor botulinum toxin injection.

Intradetrusor botulinum toxin injections were approved by the FDA for patients with refractory OAB in 2013. The toxin - derived from the fermentation of the bacterium Clostridium botulinum - impairs the ability of the smooth muscle to contract, by inhibiting the release of acetylcholine at the neuromuscular junction. The toxin is delivered into the detrusor via cystoscopic injections, and the decrease in contractions reduces OAB symptoms. The benefit generally lasts 6 to 9 months, and the effect begins to diminish as the neurons regain their ability to release neurotransmitters. ${ }^{7}$ This is generally considered to be an easy procedure. However, complications have been described, and no specific data regarding learning curves are available. As a consequence, we aimed to evaluate the learning curve of residents with no previous experience with cystoscopy to perform intradetrusor injections with botulinum toxin for the treatment of refractory OAB.

\section{Patients and Methods}

This was a retrospective study. We analyzed all consecutive women with idiopathic and refractory overactive bladder who underwent botulinum toxin bladder injections performed by residents under consultant supervision, between 2017 and 2020. OAB was defined according to The International Continence Society (ICS) as "urgency, with or without urge incontinence, usually with frequency and nocturia"1. Refractory overactive bladder was defined as persistence of symptoms despite pharmacological treatment at clinical interview. The study group also included women with mixed incontinence. Exclusion criteria were previous history of radical pelvic surgery, neurological disease, and inability to perform clean intermittent self-catheterization if necessary. Clinical assessment included a medical interview to collect clinical and obstetric history and lower urinary tract symptoms. Pelvic examination was performed according to the Pelvic Organ Prolapse Quantification system (POP-Q). ${ }^{9}$ Urodynamic evaluation comprehended uroflowmetry, cystomanometry, and a Q-tip test, as previously described. ${ }^{10}$ All procedures and definitions were conformed to the Good Urodynamic Practice Guidelines of the International Continence Society. ${ }^{11}$ Only patients without evidence of voiding dysfunction (including but not limited to postvoid residual (PVR) $>100 \mathrm{~mL}$ ) were admitted to botulinum toxin bladder injections. Incontinence severity was self-assessed with the International Consultation on Incontinence Questionnaire-Short Form questionnaire (ICIQ-SF). ${ }^{12}$ General health status was evaluated with the Euroqol EQ-5D questionnaire. ${ }^{13}$ Patients were screened for urinary tract infection with a negative urine culture. Antibiotic prophylaxis was performed 3 days before, the day of the procedure, and 2 days after with nitrofurantoin $100 \mathrm{mg} /$ die. Procedures were performed in an outpatient setting by residents, with strict mentor supervision. None of the residents had previous experience with operative cystoscopy. They familiarized themselves with the procedure by viewing videos of consultant gynecologists performing botulinum toxin bladder injections, and then assisting the consultant performing the procedure. Using a straight catheter with a catheter-tip syringe, $60 \mathrm{~mL}$ of $1 \%$ lidocaine solution was instilled into the bladder and the catheter was capped for 30 minutes before the procedure. The bladder was then drained and flushed with saline to empty any remaining lidocaine or urine and subsequently filled with $200 \mathrm{~mL}$ of saline solution. A vial of botulinum toxin containing 100 units was diluted in $10 \mathrm{~mL}$ of injectable $0.9 \%$ sodium chloride solution. The botulinum toxin was injected into the bladder wall via a rigid cystoscope at about $2 \mathrm{~mm}$ in depth, to ensure that the detrusor muscle is penetrated. The injections were distributed about $1 \mathrm{~cm}$ apart across the posterior and lateral bladder walls, sparing the trigone. A total of 20 sites were injected, each receiving $0.5 \mathrm{~mL}$ of botulinum toxin solution ( 5 units). The operative time, the number of valid injections, and the complications were noted. The operator was asked to evaluate each procedure's easiness with a 5 point Likert scale ( $1=$ very difficult, 5=very easy). Patients were asked to evaluate the perceived tolerability of the procedure on a 10 points VAS scale. Spontaneous micturition and postvoid residual were evaluated before patient discharge. In the case of positive postvoid residual, the patient was required to perform clean intermittent catheterization at home and weekly evaluation. The first follow-up visit was performed at 2 weeks if the patient was dismissed in spontaneous 
micturition, at 1 week if clean intermittent catheterization was required. During this visit, postvoid residual was checked once again. Subsequent visits were scheduled every 6 months. Follow-up visits included a clinical interview, the gynecological examination, a voiding diary compilation and the evaluation of the postvoid residual. At the first follow-up visit, the Patient Global Impression of Improvement (PGI-I), the ICIQ-SF and the Euroqol EQ5D questionnaires were collected. ${ }^{14}$ Success was defined as PGI $\leq 2$. The patients were asked to communicate when symptoms recurred to calculate the duration of efficacy.

As this was an observational retrospective analysis, and clinical management of patients was not modify by the study, it was considered exempt from Institutional Review Board (IRB) approval from local Ethics Committee of San Gerardo Hospital in Monza. The study was conducted in accordance with the Declaration of Helsinki. Written informed consent was obtained from all the patients before the procedure, as part of our protocol for operative cystoscopy. Data was entered into the database by one author and double-checked by one other author. The characteristics of the procedures (mean operative time, number of valid injections, complications, subjective easiness, perceived tolerability) and patients' outcomes (PGI-I, $\triangle \mathrm{ICIQ}-\mathrm{SF}$, need for self intermittent catheterization, duration of efficacy) were considered as markers to evaluate learning curves for each resident.

\section{Statistical Analysis}

Statistical analysis was performed using JMP version 9 (SAS, Cary, NC, USA). Data were summarized using descriptive statistics and reported as mean \pm standard deviation for continuous variables and as absolute frequency (relative frequency) for non-continuous ones. Student's $t$-test was performed to evaluate differences in questionnaire scores after the procedure compared to baseline. $\mathrm{P}<0.05$ was considered statistically significant. Trends over time in the variables analyzed were graphically interpolated by the number of procedures performed using linear lines and confidence curves. The analysis of variance $F$-test was performed. Linear intercepts of the first and last procedures were reported from linear regression. $\mathrm{F}<0.05$ was considered statistically significant.

\section{Data Availability}

The data associated with the paper are not publicly available but are available from the corresponding author on reasonable request.
Table I Baseline Symptoms and Urodynamic Findings

\begin{tabular}{|l|l|}
\hline Overactive bladder syndrome & $27(100 \%)$ \\
\hline Stress incontinence & $\mathrm{II}(40.7 \%)$ \\
\hline Voiding difficulties & $0(0 \%)$ \\
\hline ICIQ-SF & $17.8 \pm 2.6$ \\
\hline EQ-5D I-2 & $2.7 \pm 2.4$ \\
\hline EQ-5D 2-2 & $66 \pm 19$ \\
\hline Number of previous pharmacological lines & $1.5 \pm 0.8$ \\
\hline Detrusor overactivity & $21(77.7 \%)$ \\
\hline Low-compliance & $\mathrm{I}(3.7 \%)$ \\
\hline
\end{tabular}

Note: Continuous data as mean $( \pm \mathrm{SD})$. Non-continuous data as $n(\%)$.

\section{Results}

In the period of interest 27 patients underwent intravesical injection of botulinum toxin performed by three residents. Mean age was $67.3 \pm 10.1$ years, and $24(88.9 \%)$ of patients were in menopause. Among relevant comorbidities, 15 (55.6\%) women had hypertension, 7 (25.9\%) had glaucoma, and 3 (11.1\%) had hiatal hernia. Baseline lower urinary tract symptoms and urodynamics findings are reported in Table 1. Of note, detrusor overactivity was identified in $21(77.7 \%)$ patients, while 1 (3.7\%) woman had a low-compliant bladder. Most women (55.6\%) had at least two previous pharmacological treatments, discontinued for either inefficacy, collateral effects, or costs. The mean duration of oral pharmacological management was $10.4 \pm 9.5$ months. In all but three patients, the indication for botulinum toxin therapy was refractory OAB. However, in three women there was a coexistence of contraindications for both antimuscarinic and beta-3-agonist drugs and underwent botulinum toxin injections after the failure of behavioural management. Procedures characteristics are summarized in Table 2. Notably, only a grade 1 Clavien-Dindo complication occurred. This

Table 2 Operative Data

\begin{tabular}{|l|l|}
\hline Operative time & $13 \pm 3$ \\
\hline Number of valid injections & $20 \pm 0$ \\
\hline Complications & $1(3.7 \%)$ \\
\hline Perceived easiness & $1.5 \pm 0.8$ \\
\hline Need for self intermittent catheterization & $0(0 \%)$ \\
\hline
\end{tabular}

Note: Continuous data as mean $( \pm S D)$. Non-continuous data as $n(\%)$. 
Table 3 Outcomes

\begin{tabular}{|l|c|l|}
\hline Subjective success & $25(92.6 \%)$ \\
\hline $\begin{array}{l}\text { Duration of efficacy } \\
\text { (months) }\end{array}$ & \multicolumn{2}{|c|}{$8.0 \pm 4.3$} \\
\hline PGI & \multicolumn{2}{|c|}{$1.4 \pm 0.9$} \\
\hline Urinary retention & $0.8 \pm 4.1$ & $(p<0.0001)^{*}$ \\
\hline ICIQ-SF & $1.9 \pm 1.6$ & $(p=0.15)^{*}$ \\
\hline EQ-5D I-2 & $76 \pm 18$ & $(p=0.04)^{*}$ \\
\hline EQ-5D 2-2 & \multicolumn{2}{|c|}{$0(0 \%)$} \\
\hline
\end{tabular}

Note: Continuous data as mean ( \pm SD). Non-continuous data as n $(\%) *$ compared to preprocedural values.

was a 2-3 cm growing suburothelial hematoma whose expansion was halted applying mild bimanual pressure on the bladder for a few minutes. Treatment outcomes are shown in Table 3. None of the patients had urinary retention, and the global impression of improvement was very satisfactory, with PGI-I scoring $1.4 \pm 0.9$. Both ICIQSF and EQ-5D 2-2 were statistically improved ( $<<0.00001$ and $\mathrm{p}=0.04$ respectively) compared to preoperative scores. None of the patients had urinary tract infections. The mean duration of efficacy as reported by patients resulted to be $8.0 \pm 4.3$ months. Each resident (A, B, C) performed nine procedures. Statistically significant trends of variables from the first to the last procedure for at least one resident are reported in Figure 1. Among procedural variables, a positive effect of the learning curve was observed only for operative time, subjective easiness, and perceived tolerability. On the contrary, the number of valid injections and complications were not affected by physician experience. None of the considered outcomes was significantly influenced by the learning curve.
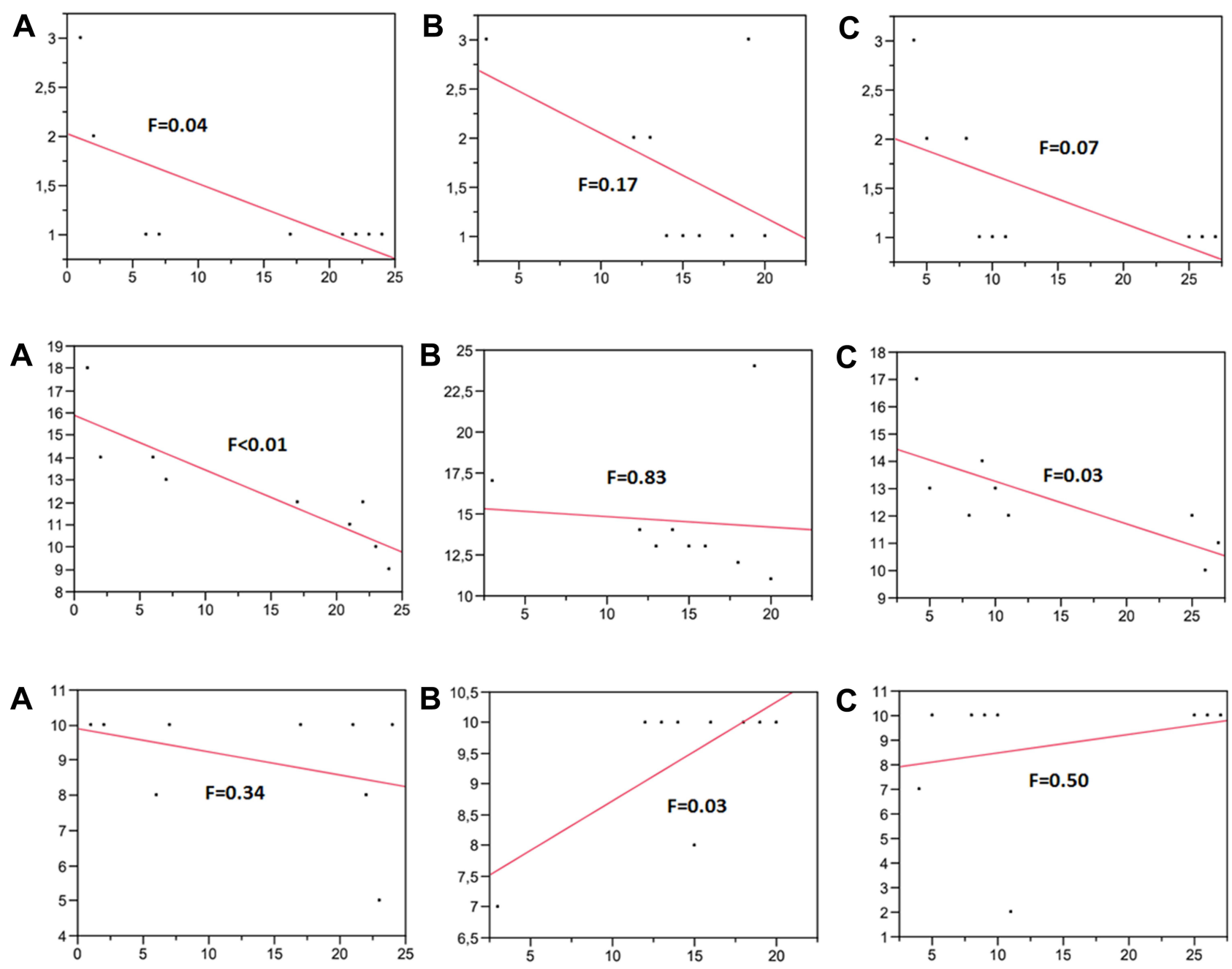

Figure I Linear regressions for statistically significant variables, for each resident (A-C). I= Subjective easiness on 5-point Likert scale; $X$-axis: sequential number of procedures; Y-axis: score. 2= Operative time. X-axis: sequential number of procedures; Y-axis: minutes. 3= Perceived tolerability on I0-points VAS scale; X-axis: sequential number of procedures; Y-axis: score. 


\section{Discussion}

In medicine, many factors contribute to whether a physician can perform a particular procedure, including the extent of their medical knowledge, specific training as well as the degree of manual skills they possess. Recently, there is a great interest in the evaluation of the impact of these factors in terms of success and complications of medical procedures. Learning curves graphically represent the impacts of the repetitive task over a defined period, in terms of specific outcomes, such as operative time, complications, and cure rates. The concept of a learning curve could be useful to define what is an acceptable level of performance for a given procedure. Boundaries could be used to evaluate the trainees' performance and consider actions such as further education, or re-training. They may also help to evaluate the efficacy of strategies aimed to shorten learning curves and improve outcomes even at the very first procedures, such as close mentorship or virtual simulators. Despite intradetrusor botulinum toxin injections is a well defined second-line treatment option for patients with refractory $\mathrm{OAB}$, no data are available about its learning curve. With this study, we aimed to evaluate the learning curve of residents with no previous experience with cystoscopy to perform intradetrusor injections with botulinum toxin for the treatment of refractory OAB. We found that intradetrusor botulinum toxin injection is a procedure characterized by high efficacy and very low complications even at the very beginning of the learning curve. Moreover, the impact of physician experience seems to be limited to periprocedural variables - such as operative time, subjective easiness, and patient discomfort - without affecting outcomes.

In our series, we found a subjective cure rate of $92.6 \%$, with significant improvements of perceived $\mathrm{OAB}$ severity and quality of life according to ICIQ-SF and EQ-5D questionnaires compared to baseline. Our cure rate is consistent with the upper values of the reported range, which varies from $36 \%$ to $89 \%$ depending on the considered definition of efficacy and assessment tool used. ${ }^{15}$ In our study benefits lasted for a mean of 8 months, which is in the reported range of 4-10 months of efficacy after single treatment reported in the literature. ${ }^{15}$ In our series, we registered only one $(3.7 \%)$ complication, which was a $2-$ $3 \mathrm{~cm}$ suburothelial hematoma (grade 1 according to Clavien-Dindo classification). This confirmed the safety of the procedure, which is characterized by a low incidence of local adverse effects such as bruising and/or local small hematoma, pain at the injection site, and pelvic pain. ${ }^{15}$ Specifically, our data is concordant to the rate of haematuria reported in the literature, ranging between $3.2 \%$ and $5.0 \%{ }^{16}$ No systemic adverse effects such as hyposthenia or flu-like symptoms were registered in our series, but these are more frequently reported for higher doses of botulinum toxin. ${ }^{17}$ Interestingly, none of the patients had positive PVR at discharge and needed clean intermittent self-catheterization. Rates of need for selfcatheterization after botulinum toxin bladder injection are extremely variable, ranging from $4 \%$ to $45 \% .{ }^{15}$ However, voiding dysfunction is a dosage-dependent adverse effect. In our series, low botulinum toxin dosage and accurate patient selection may explain the absence of patients with positive PVR after the procedure.

We also found that the impact of the learning curve is limited, and regards variables with limited clinical importance, such as operative time and perceived easiness. The most relevant parameter, which was found to be related to surgeon experience, was the perceived tolerability evaluated by the patients, which improved from the first to the last procedure in one resident learning curve. Interestingly, complications, cure rates, quality of life, need for self intermittent catheterization, and duration of efficacy resulted satisfactory and unmodified by residents' experience.

This means that intravesical botulinum toxin injections can be performed safely and efficiently even during the initial learning curve under close mentorship. Up-to-date there are no studies about the learning curve for this procedure. However, there is some evidence about the role of the learning curve for other operative cystoscopic procedures, which seems to be negligible. Yamaçake et al evaluated the learning curve for transurethral resection of the prostate among urology residents and found that operative time, the weight of resected tissue, and the incidence of capsular lesions improve after the performance of 10 procedures. ${ }^{18}$ Similarly, Feng et al analyzed the learning curve of one single surgeon with transurethral plasmakinetic enucleation/resection of prostate and found excellent safety and efficiency even at the beginning of the learning curve of the surgeon. ${ }^{19}$

Interestingly, learning curves in urogynecology are often based on performances of a single operator, thus limiting the generalization of the data. ${ }^{20,21}$ Moreover, often studies assessing the learning curve in cystoscopy are based on training on virtual simulators. ${ }^{22,23}$ In our series, data were evaluated for three different residents, 
making our findings more reproducible. Another strength of our study is the real-life setting, which makes data more reliable. Moreover, several parameters - including perioperative data, complications, and outcomes - have been considered as possible variables influenced by residents' experience. Limitations include the retrospective design and the limited number of procedures considered.

\section{Conclusion}

Intradetrusor botulinum toxin injection for the treatment of refractory $\mathrm{OAB}$ is a procedure characterized by high efficacy and very low complications even at the very beginning of the learning curve when performed under proper mentorship.

\section{Author Contributions}

All authors made a significant contribution to the work reported, whether that is in the conception, study design, execution, acquisition of data, analysis and interpretation, or in all these areas; took part in drafting, revising or critically reviewing the article; gave final approval of the version to be published; have agreed on the journal to which the article has been submitted; and agree to be accountable for all aspects of the work.

\section{Funding}

There is no funding to report.

\section{Disclosure}

The authors report no conflicts of interest in this work.

\section{References}

1. Abrams P, Cardozo L, Fall M, et al. The standardisation of terminology of lower urinary tract function: report from the standardisation sub-committee of the International Continence Society. Neurourol Urodyn. 2002;21(2):167-178. doi:10.1002/nau.10052

2. Stewart WF, Van Rooyen JB, Cundiff GW, et al. Prevalence and burden of overactive bladder in the United States. World J Urol. 2003;20(6):327-336. doi:10.1007/s00345-002-0301-4

3. Yamaguchi O, Honda K, Nomiya M, et al. Defining overactive bladder as hypersensitivity. Neurourol Urodyn. 2007;26(Suppl):904-907. doi:10.1002/nau.20482

4. Frigerio M, Manodoro S, Cola A, Palmieri S, Spelzini F, Milani R. Risk factors for persistent, de novo and overall overactive bladder syndrome after surgical prolapse repair. Eur $J$ Obstet Gynecol Reprod Biol. 2019;233:141-145. doi:10.1016/j.ejogrb.2018.12.024

5. Manodoro S, Barba M, Locatelli L, Palmieri S, Marino G, Frigerio M. Urodynamic predictors of de novo overactive bladder after single-incision sling. Int J Gynaecol Obstet. 2020;148 Suppl 2:29. doi:10.1002/ijgo.13045

6. Hashim H, Abrams P. Is the bladder a reliable witness for predicting detrusor overactivity? J Urol. 2006;175(1):191-195. doi:10.1016/ S0022-5347(05)00067-4
7. Jayarajan J, Radomski SB. Pharmacotherapy of overactive bladder in adults: a review of efficacy, tolerability, and quality of life. Res Rep Urol. 2013;6:1-16. doi:10.2147/RRU.S40034

8. Imran M, Najmi AK, Tabrez S. Mirabegron for overactive bladder: a novel, first-in-class b3-agonist therapy. Urol J. 2013;10 (3):935-940.

9. Bump RC, Mattiasson A, Bø K, et al. The standardization of terminology of female pelvic organ prolapse and pelvic floor dysfunction. Am J Obstet Gynecol. 1996;175(1):10-17. doi:10.1016/S00029378(96)70243-0

10. Manodoro S, Spelzini F, Frigerio M, Nicoli E, Verri D, Milani R. Is occult stress urinary incontinence a reliable predictive marker? Female Pelvic Med Reconstr Surg. 2016;22(4):280-282. doi:10.1097/SPV.0000000000000272

11. Schäfer W, Abrams P, Liao L, et al. Good urodynamic practices: uroflowmetry, filling cystometry, and pressure-flow studies. Neurourol Urodyn. 2002;21(3):261-274. doi:10.1002/nau.10066

12. Tubaro A, Zattoni F, Prezioso D, et al. Italian validation of the international consultation on incontinence questionnaires. BJU Int. 2006;97(1):101-108. doi:10.1111/j.1464-410X.2006.05885.x

13. Hurst NP, Kind P, Ruta D, Hunter M, Stubbings A. Measuring health-related quality of life in rheumatoid arthritis: validity, responsiveness and reliability of EuroQol (EQ-5D). $\mathrm{Br} J$ Rheumatol. 1997;36(5):551-559. doi:10.1093/rheumatology/36.5.551

14. Srikrishna S, Robinson D, Cardozo L. Validation of the Patient Global Impression of Improvement (PGI-I) for urogenital prolapse. Int Urogynecol J. 2010;21(5):523-528. doi:10.1007/s00192-0091069-5

15. Apostolidis A, Dasgupta P, Denys P, et al.; European Consensus Panel. Recommendations on the use of botulinum toxin in the treatment of lower urinary tract disorders and pelvic floor dysfunctions: a European consensus report. Eur Urol. 2009;55(1):100-119. doi:10.1016/j.eururo.2008.09.009

16. Ghei M, Maraj BH, Miller R, et al. Effects of botulinum toxin B on refractory detrusor overactivity: a randomized, double-blind, placebo controlled, crossover trial. J Urol. 2005;174(5):1873-1877. doi:10.1097/01.ju.0000177477.83991.88

17. Ruffion A, Capelle O, Paparel P, Leriche B, Leriche A, Grise P. What is the optimum dose of type A botulinum toxin for treating neurogenic bladder overactivity? BJU Int. 2006;97(5):1030-1034. doi:10.1111/j.1464-410X.2006.06091.x

18. Yamaçake KG, Nakano ET, Soares IB, Cordeiro P, Srougi M, Antunes AA. Analysis of the learning curve for transurethral resection of the prostate. Is there any influence of musical instrument and video game skills on surgical performance? Turk J Urol. 2015;41 (3):132-137. doi:10.5152/tud.2015.01112

19. Feng L, Song J, Zhang D, Tian Y. Evaluation of the learning curve for transurethral plasmakinetic enucleation and resection of prostate using a mentor-based approach. Int Braz J Urol. 2017;43(2):245-255. doi:10.1590/s1677-5538.ibju.2016.0237

20. Spelzini F, Frigerio M, Regini C, Palmieri S, Manodoro S, Milani R. Learning curve for the single-incision suburethral sling procedure for female stress urinary incontinence. Int J Gynaecol Obstet. 2017;139 (3):363-367. doi:10.1002/ijgo.12317

21. Song R, He X, Chang Y, et al. Learning curve for the tension-free vaginal tape-obturator procedure for female stress urinary incontinence: a prospective single-surgeon study. J Endourol. 2020;34 (2):209-214. doi:10.1089/end.2019.0571

22. Schout BM, Muijtjens AM, Hendrikx AJ, et al. Acquisition of flexible cystoscopy skills on a virtual reality simulator by experts and novices. BJU Int. 2010;105(2):234-239. doi:10.1111/j.1464410X.2009.08733.x

23. Kruck S, Bedke J, Hennenlotter J, et al. Virtual bladder tumor transurethral resection: an objective evaluation tool to overcome learning curves with and without photodynamic diagnostics. Urol Int. 2011;87(2):138-142. doi:10.1159/000328218 


\section{Publish your work in this journal}

The International Journal of Women's Health is an international, peerreviewed open-access journal publishing original research, reports, editorials, reviews and commentaries on all aspects of women's healthcare including gynecology, obstetrics, and breast cancer. The manuscript management system is completely online and includes a very quick and fair peer-review system, which is all easy to use. Visit http://www.dovepress.com/testimonials.php to read real quotes from published authors. 